\title{
Modélisation de la diffusion des gènes Holstein à l'intérieur de la population bovine Pie Noir Française
}

\author{
J.J. COLLEAU et D. TANGUY \\ I.N.R.A., Station de Génétique quantitative et appliquée, \\ Centre de Recherches zootechniques, F 78350 Jouy-en-Josas
}

\begin{abstract}
Résumé
L'utilisation de reproducteurs Holstein est actuellement très importante en race bovine Française Frisonne, en raison de leur supériorité laitière affirmée. La cinétique de la transmission de leurs gènes aux animaux commerciaux est étudiée dans cet article. La technique générale adoptée consiste à utiliser les méthodes de modélisation matricielle développées pour les schémas de sélection en race pure mais en fractionnant la population en sous-populations correspondant à des classes de proportion de gènes Holstein, de manière à traiter des matrices de taille constante et raisonnable. Dans ce cadre, des algorithmes de calcul approché sont proposés pour évaluer le résultat du croisement entre populations hétérogènes et pour évaluer le résultat d'une sélection sur une population hétérogène.

L'application de la méthode et la prise en compte des décisions de sélection déjà prises en France dans le passé permet d'évaluer à environ $35 \mathrm{p}$. 100 la proportion de gènes Holstein dans la population des veaux Pie Noir nés en France en 1983. En ce qui concerne le futur, 2 hypothèses extrêmes sont envisagées. En cas d'arrêt pur et simple des importations Holstein, la fréquence des gènes Holstein augmentera encore pendant environ 5 ans pour se stabiliser aux alentours de 50 p. 100. En cas de testage systématique de taureaux Holstein purs, ce qui exige la continuation des importations, l'absorption sera relativement rapide puisque dans 15 ans, les jeunes veaux de la population commerciale possèderaient alors 85 p. 100 de gènes Holstein. Ces valeurs dépendent assez peu des paramètres étudiés (différence initiale de niveaux génétiques entre la France et l'Amérique du Nord, efficacité future des programmes de sélection dans les 2 pays). Ce n'est pas le cas pour les évolutions de niveaux génétiques laitiers. Pour les 2 types de calcul (fréquences et niveaux génétiques laitiers), les résultats obtenus sont très semblables, que l'on considère 10 ou 30 classes de piuportion de gènes Holstein.
\end{abstract}

Mots clés : Croisement, migration, modèle mathématique.

\section{Summary}

A model for the diffusion of Holstein genes within the French Friesian cattle population

The current importance of using Holstein sires to upgrade French Friesian herds is due to the superior dairying qualities of the Holstein. We have studied the spread of Holstein genes among commercial cattle using methods of matrix modelling developed for selection programmes in pure breeds, with the modification that our population was divided into subgroups, according to the proportion of Holstein genes they contained, and in such a way that the different matrices produced were of constant and restricted size. As a result, we propose algorithmes which can be used to estimate the result of crossbreeding heterogeneous populations, and to evaluate the effect 
of selection on one such population. Making allowance for selection policies applied in the past, this approach predicts that there will be about 35 p. 100 Holstein genes among French Friesian calves born in 1983. Concerning future developments, we consider 2 extreme cases. If imports of Holstein bulls are to end immediately, we predict that the frequency of Holstein genes would continue to increase for the next 5 years and would then stabilize at around 50 p. 100. On the other hand, if the use of purebred Holstein sires were to remain a priority, necessitating continued imports, the dissemination of Holstein genes would progress relatively rapidly until, in 15 years time, commercial calves would carry an estimated 85 p. 100 of Holstein genes.

The parameters studied, such as the genetic difference between France and the U.S. or the future efficiency of the selection schemes in both countries, have little influence on the predictions of Holstein gene diffusion but a large influence on the predictions of breeding values for milk production. Both kinds of calculation (frequencies or breeding values) considering 10 or 30 classes for the proportion of Holstein genes, lead to very similar results.

Key words : Crossbreeding, migration, mathematic model.

\section{Introduction}

On note depuis une bonne dizaine d'années une pénétration régulière du rameau Pie Noir Holstein dans les populations Pie Noir européennes (CunNINGHAM, 1978 ; Krausslich \& Jongeling, 1978 ; Bech-ANDERSEN, 1982 ; Southgate, 1982 ; Milk Marketing Board, 1982 ; COLlEAU et al., 1982). La cause essentielle de ce bouleversement génétique est la très grande supériorité génétique laitière initiale du rameau Holstein sur les populations européennes. Les estimations communément rencontrées sont de l'ordre de 1000 à $1300 \mathrm{~kg}$ de lait par lactation soit environ 2 à 2,5 écarts-types génétiques (WITT et al., 1971 ; ERNST et al., 1973 ; JOURNET et al., 1973 ; KRAUSSLICH, 1974 ; Minkema, 1974 ; OldEnBroek, 1974, 1979, 1980, 1983a ; LEROY, 1977 ; Stolzman et al., 1981 ; Turton, 1981 ; Politiek et al., 1982). Comme l'a montré un certain nombre d'études techniques, ce croisement s'accompagne d'effets défavorables dans le domaine de la production de viande et de la reproduction (WITT et al., 1971 ; JoURNET et al., 1973 ; ERNST et al., 1973 ; REgIs, 1979, 1980 ; JoNGELING \& NiEDHAVES, 1979 ; PhilipPson et al., 1981 ; STOLzMAN et al., 1981 ; Southgate, 1982 ; BeCH-ANDERSEN, 1982b ; Oldenbroek, 1982, 1983a). Cependant, d'un point de vue économique, ces effets sont loin d'annuler la plus-value entraînée par l'amélioration de la production laitière (Dubis, 1979 ; Oldenbroek, 1983b). De ce fait, le degré définitif de croisement avec la Holstein sera très important. La situation française est comparable à celle des autres pays de l'Europe de l'Ouest. Le phénomène est encore amplifié par la progression propre de la Pie Noir au détriment des races mixtes nationales. Les questions posées à long terme concernent la structure même de l'élevage bovin, notamment la place quantitative à donner aux races mixtes et aux races à viande. Pour que notre pays puisse s'adapter de façon adéquate à cette situation, il paraît essentiel de disposer de bons outils de prévision.

L'objet de cet article est ainsi d'évaluer quantitativement pour le présent et pour le futur, la diffusion des gènes Holstein dans la population des animaux commerciaux. Cette évaluation est la condition indispensable à une appréciation réaliste des performances bouchères actuelles et futures de la race Pie Noir, qui est de loin la race laitière démographiquement prédominante dans notre pays. L'étude de la diffusion des gènes Holstein permet également de quantifier l'accélération du progrès génétique laitier, qui est le but recherché par le croisement. 


\section{Matériel et méthodes}

\section{A. Contexte technique de la holsteinisation}

\section{Existence de deux composantes du processus de la holsteinisation}

Ce processus de croisement s'effectue dans le cadre des programmes de sélection normaux en race Pie Noir. Nous ferons l'approximation que ces programmes sont axés avant tout sur les caractères de production laitière. Ces programmes contribuent donc à diffuser les gènes Holstein existant déjà dans la population.

La diffusion de ces gènes est en outre accélérée par les importations d'animaux Holstein. Le fait est particulièrement net pour les jeunes taureaux mis en testage. De ce fait, les calculs prédictifs doivent en tenir compte. En revanche, les importations de femelles Holstein peuvent être considérées comme négligeables.

\section{Description du programme de sélection}

et des paramètres génético-démographiques utilisés

a) Le critère de sélection utilisé est la quantité moyenne de matière utile (MU), telle que la définissent Poutous et al. (1980) soit $\mathrm{MU}=1 / 2(\mathrm{MG}+1,21 \mathrm{MP})$ avec MG = quantité de matière grasse et $\mathrm{MP}=$ quantité de matière protéique. Ce critère permet en race pure l'augmentation maximum de production laitière compatible avec un maintien génétique des taux butyreux et protéiques.

b) L'écart-type génétique intra souche est de $17 \mathrm{~kg}$ MU (BonAITI \& Mocouot, 1982 ; MoCQUOT \& BonAITI, 1981). Il est en toute rigueur légèrement plus faible $(15 \mathrm{~kg})$ à l'intérieur des souches européennes.

c) Les pères à taureaux sont choisis parmi la fraction $C_{p}=0,03$ supérieure en MU de la population des taureaux testés en France, le coefficient de détermination de l'index étant égal à 0,85 .

d) Les mères à taureaux sont choisies parmi la fraction $C_{M}=0,03$ supérieure en MU de la population des vaches nées en France et ayant au moins 3 lactations, le coefficient de détermination de l'index étant égal à 0,50 .

e) Le testage des taureaux a lieu à 1,5 an. L'index des taureaux est calculé à 5,5 ans. On suppose que l'ensemble des taureaux de testage réalise 15 p. 100 des inséminations totales sur le cheptel femelle.

f) Les taureaux de service sont choisis dans la fraction $\mathrm{C}_{\mathrm{s}}=0,50$ supérieure en MU de la population des taureaux de testage. Ils sont supposés être utilisés ensuite sans interruption et de façon régulière pendant 2 ans, indépendamment de leur classe génétique.

g) Les vaches sont supposées être utilisées pendant 8 lactations au maximum après un premier vêlage à 3 ans, les vaches frisonnes ayant plus de 8 lactations au contrôle laitier constituant seulement 2 p. 100 des effectifs. Les fréquences des différentes lactations sont celles du contrôle laitier soit 27 p. 100,22 p. 100,17 p. 100,13 p. 100 , 9 p. 100,6 p. 100,4 p. 100,2 p. 100 . La réforme est supposée s'effectuer indépendamment du niveau laitier, ce qui est une simplification évidente. 
h) La pression de sélection sur ascendance effectuée sur les génisses de renouvellement est supposée être négligeable en raison de la faible prolificité de l'espèce bovine et en raison des importants besoins de renouvellement dans cette espèce.

\section{Les importations Holstein}

Les tableaux 1 et 2 donnent la composition génétique des séries de taureaux de testage et de service connues au moment du début des calculs correspondant à cet article. Ces tableaux matérialisent les décisions passées de sélection. Le vrai moteur du croisement Holstein consiste en l'importation de jeunes taureaux Holstein (jusqu'à 75 p. 100 des taureaux Holstein purs mis au testage), comme il apparaît au tableau 1. L'accroissement de la proportion d'inséminations Holstein n'est que la conséquence de ce fait puisque dans notre pays les importations directes de semence de reproducteurs Holstein sont très faibles (moins de 1 p. 100).

\section{B. Description du modèle utilisé}

1. Assimilation de la proportion de gènes Holstein à une variable discrète

Dans cette étude, la proportion de gènes Holstein des différents individus de la population Pie Noir Française est considérée comme une variable discrète.

Cette manière de procéder présente comme avantage de permettre d'établir les relations de récurrence entre années avec des matrices ou vecteurs de dimensions constantes, ce qui est particulièrement commode car le nombre de types génétiques réellement différents croît de manière exponentielle au cours du temps. Les traitements mathématiques seraient alors très lourds. C'est cette lourdeur que nous nous sommes proposés d'éliminer grâce au concept de discrétisation. Nous nous proposons d'évaluer par la suite la perte de précision ainsi entraînée.

On constitue $\mathrm{p}$ classes équidistantes de proportion de gènes Holstein, d'amplitude $1 / \mathrm{p}$. La jième classe génétique regroupe les animaux dont la fraction de gènes Holsteins s'étale de $\frac{j-1}{p}$ à $\frac{j}{p}$, la valeur centrale étant $h_{j}=\frac{j-0,5}{p}$.

Une catégorie d'animaux déterminée, par exemple les taureaux de testage nés une année donnée, est représentée par un vecteur ligne $V$ de fréquences. Si $j$ est l'indice classe génétique, on a alors $\sum_{j=1}^{p} v_{j}=1$. Le vecteur $V^{*}$ est le vecteur correspondant des niveaux génétiques, de terme $\mathrm{v}_{\mathrm{j}}{ }^{*}$.

\section{Expression des résultats de l'accouplement au hasard d'une population mâle} et d'une population femelle

\section{a) Considérations générales}

D’une manière générale, la population mâle peut être représentée par un vecteur ligne $\mathrm{V}(1, \mathrm{p})$ de fréquences et un vecteur $\mathrm{V}^{*}$ correspondant de niveaux génétiques. La population femelle est décrite par les vecteurs $W$ et $W^{*}$ de même dimension. La population des descendants est représentée par le vecteur $\mathrm{Z}$ et $\mathrm{Z}^{*}$ de même dimension que les vecteurs des populations parentales. 


\section{TABLEAU 1}

Répartition génétique des taureaux de testage.

Genetic distribution of the young bulls.

\begin{tabular}{|c|c|c|c|c|c|c|c|}
\hline \multirow{2}{*}{$\begin{array}{c}\text { Année } \\
\text { de naissance }\end{array}$} & \multicolumn{6}{|c|}{ TYPE GÉNÉTIQue ( $\%$ gènes Holstein) } & \multirow{2}{*}{$\begin{array}{l}\text { Proportion de } \\
\text { Holstein purs } \\
\text { importés (\%) }\end{array}$} \\
\hline & $0 \%$ & $25 \%$ & $50 \%$ & $75 \%$ & $100 \%$ & $\%$ moyen & \\
\hline 68 & 93 & & 2 & & 5 & 6 & 18 \\
\hline 69 & 92 & & & & 8 & 8 & 40 \\
\hline 70 & 85 & 1 & 3 & & 11 & 13 & 40 \\
\hline 71 & 73 & 1 & 9 & & 16 & 21 & 60 \\
\hline 72 & 63 & 1 & 11 & 1 & 25 & 31 & 75 \\
\hline 73 & 57 & 3 & 13 & & 27 & 34 & 72 \\
\hline 74 & 57 & 1 & 10 & 1 & 31 & 37 & 76 \\
\hline 75 & 51 & 4 & 17 & 3 & 26 & 37 & 65 \\
\hline 76 & 42 & 4 & 14 & 8 & 32 & 46 & 59 \\
\hline 77 & 31 & 4 & 19 & 11 & 35 & 54 & 57 \\
\hline 78 & 10 & 2 & 22 & 9 & 57 & 75 & 75 \\
\hline 79 & 5 & 1 & 22 & 20 & 51 & 78 & 66 \\
\hline 80 & 3 & 1 & 18 & 33 & 45 & 79 & 38 \\
\hline
\end{tabular}

Tableau 2

Répartition génétique des taureaux de service.

Genetic distribution of the service bulls.

\begin{tabular}{c|l|c|c|c|c|c}
\hline \hline \multirow{2}{*}{ Année de service } & \multicolumn{5}{|c}{ TYPE GENÉTIQUE (\% gènes Holstein) } \\
\cline { 2 - 7 } & $0 \%$ & $25 \%$ & $50 \%$ & $75 \%$ & $100 \%$ & $\%$ moyen \\
\hline & & & & & & \\
68 & 99,6 & & & & 0,4 & 0,4 \\
69 & 99 & & & & 1 & 1 \\
70 & 98 & & & & 2 & 2 \\
71 & 96,5 & & & & 3,5 & 3,5 \\
72 & 95 & & & & 5 & 5 \\
73 & 94 & & 1 & & 6 & 6 \\
74 & 96 & & & & 4 & 4 \\
75 & 91 & & 2 & & 7 & 8 \\
76 & 88 & & 2 & & 10 & 11 \\
77 & 83 & & 2 & & 19 & 21 \\
78 & 77 & 0,5 & 3 & & 30 & 34 \\
79 & 61 & 1 & 8 & & 39 & 45 \\
\hline 0 & 49 & & 11 & & & \\
\hline
\end{tabular}


La production de gènes Holstein chez les produits issus d'un accouplement entre un mâle appartenant à la classe $\mathrm{j}^{\prime}$ et une femelle de la classe $\mathrm{j}^{\prime \prime}$ est égale à :

$$
\frac{1}{2}\left(h_{j^{\prime}}+h_{j^{\prime \prime}}\right)=\frac{1}{p}\left[\frac{1}{2}\left(j^{\prime}+j^{\prime \prime}\right)-0,5\right]
$$

Il y a donc autant de types de produits que de valeurs différentes de la somme $\mathrm{s}=\mathrm{j}^{\prime}+\mathrm{j}^{\prime \prime}$. Cette somme varie de 2 à $2 p$, ce qui donne naissance à $2 p-1$ valeurs distinctes. La cohérence exige que ces $2 p-1$ classes de produits soient regroupées en $\mathrm{p}$ classes. Les principes du regroupement sont les suivants :

- quand $s=2 r$ (somme paire), la proportion de gènes Holstein des produits est égale à $\frac{1}{\mathrm{p}}(\mathrm{r}-0,5)$. Les produits sont attribués à la rème classe puisque cette valeur est égale à $\mathrm{h}_{\mathrm{r}}$;

- quand $s=2 r+1$ (somme impaire), la proportion de gènes Holstein est égale à $\frac{1}{p}(r+0,5-0,5)$ soit aussi $\frac{1}{2 p}(r-0,5)+\frac{1}{2 p}(r+1-0,5)$.

On attribue la moitié des produits à la rème classe et l'autre moitié à la $r+1^{\text {ème }}$ classe.

Cette méthode entraîne une nouvelle perte de précision. Le fait de procéder à des équivalences sur les catégories les plus proches minimise toutefois cette perte.

b) Calcul du vecteur $\mathrm{Z}$ des fréquences :

On crée une matrice bande $\Delta\left(\mathrm{V}^{\prime} \mathrm{W}\right)$ à partir du produit $\mathrm{V}^{\prime} \mathrm{W}$ :

$$
\Delta=\left(0 \longdiv { \mathrm { V } ^ { \prime } \mathrm { W } } \mathrm { O }\right)
$$

Cette matrice a pour dimensions (p, 2p - 1).

La sommation sur les lignes par colonne $1^{\prime} . \Delta$ donne les fréquences pour chacune des $2 \mathrm{p}-1$ classes.

Le vecteur $\mathrm{Z}$ est obtenu en postmultipliant $1^{\prime} . \Delta$ par une matrice $\mathrm{A}$ de dimension $(2 \mathrm{p}-1, \mathrm{p})$ définie par :

$$
\begin{aligned}
& a_{k j}=1 \text { pour } k=2 j-1 \\
& a_{k j}=0,5 \text { pour }\left\{\begin{array}{l}
k=2 j-2 \\
k=2 j
\end{array}\right. \\
& a_{k j}=0 \text { dans les autres cas. }
\end{aligned}
$$

En conclusion, on a :

$$
\underset{(1, p)}{Z}=\underset{(1, p)}{1^{\prime}} \cdot \underset{(p, 2 p-1)}{\Delta\left(V^{\prime} W\right)} \cdot \underset{(2 p-1, p)}{A}
$$

Le terme général s'écrit :

$$
\mathrm{z}_{\mathrm{j}}=\sum_{\mathrm{k}=1}^{2 \mathrm{p}-1}\left(\sum_{\mathrm{j}=1}^{\mathrm{p}} \delta_{\mathrm{jk}}\right) \mathrm{a}_{\mathrm{kj}}
$$

où $\delta_{\mathrm{jk}}$ désigne le terme général de $\Delta$. 
c) Calcul du vecteur $Z^{*}$ des niveaux génétiques

On crée la matrice bande $\Delta^{*}\left(\mathrm{~V}^{*} \cdot 1^{\prime}+1 \mathrm{~W}^{*}\right)$ à partir de la demi-somme de chacun des éléments de $\mathrm{V}^{*}$ avec chacun des éléments de $\mathrm{W}^{*}$ :

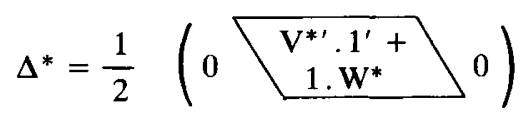

Cette matrice a pour dimension $(p, 2 p-1)$ et a pour terme général $\delta_{j k}^{*}$ avec $j$ variant de 1 à $\mathrm{p}$ et $\mathrm{k}$ variant de 1 à $2 \mathrm{p}-1$.

En se servant des matrices $\Delta$ et $\Delta^{*}$, il est possible de calculer pour chacune des $2 p-1$ classes les totaux marginaux et les fréquences marginales. Ces $2 p-1$ classes peuvent être ramenées à $\mathrm{p}$ classes suivant le procédé décrit au paragraphe 1 .

Le terme général $z_{j}^{*}$ de $Z^{*}$ s'écrit alors :

$$
z_{j}^{*}=\frac{1}{z j} \sum_{k=1}^{2 p}-1\left(\sum_{j=1}^{p} \delta_{j k}^{*} \delta_{j k}\right) a_{k j}
$$

L'expression matricielle de $\mathrm{Z}^{*}$ avec les opérateurs classiques de l'algèbre matricielle est très lourde. Pour cette raison, nous ne la donnerons pas.

Elle est en revanche simple à établir si l'on admet l'existence d'opérations spéciales (division terme à terme...) telles qu'il en existe en langage APL, langage de programmation dans lequel ont été exécutées les applications numériques.

\section{3) Expression des résultats d'une sélection sur une population}

On dispose d'une population décrite par les vecteurs $\mathrm{X}$ et $\mathrm{X}^{*}$ et l'on veut connaître les vecteurs $\mathrm{Y}$ et $\mathrm{Y}^{*}$ correspondant aux populations sélectionnées.

On suppose que la sélection s'effectue par troncature sur un index dont le coefficient de détermination est égal à $\mathrm{CD}$ et qui a été établi sans tenir compte de l'existence des différentes catégories génétiques. La fraction d'animaux conservés correspond à $\mathrm{c}$.

Dans ce cas, le vecteur $\mathrm{I}(1, \mathrm{p})$ des index moyens est égal à $\mathrm{I}=\mathrm{Cte}+\mathrm{CD} \mathrm{X}^{*}$. L'annulation de la constante ne change rien aux calculs ultérieurs soit $\mathrm{I}^{*}=\mathrm{CD}$. $\mathrm{X}^{*}$.

L'écart-type des index pour une catégorie génotypique donnée est égal à $\sqrt{\mathrm{CD}} \sigma$ où $\sigma$ désigne l'écart-type génétique laitier intragénotype.

On fait alors le changement de variable $\alpha^{*}=\frac{I^{*}}{\sqrt{\mathrm{CD}} \sigma}$ tel que les écarts-types intrapopulation soient égaux à 1 , ce qui permet d'utiliser les tables de la loi normale.

La position du seuil de troncature est définie, en posant $P=1-\Phi$ où $\Phi$ est la fonction de répartition de la loi normale, par $\alpha_{0}{ }^{*}$ tel que :

$$
\sum_{j} x_{j} P\left(\alpha_{o}^{*}-\alpha_{j}^{*}\right)=c
$$

où $x_{j}$ est la proportion initiale d'animaux du génotype $\mathrm{j}, \mathrm{P}\left(\alpha_{\mathrm{o}}{ }^{*}-\alpha_{\mathrm{j}}{ }^{*}\right)$ celle d'animaux sélectionnés dans le génotype $\mathrm{j}$ et $\mathrm{c}$ la fraction globale d'animaux sélectionnés. 
Cette équation n'est pas linéaire et est résolue par itération. A la $\mathbf{n}+1$ ième itération, on a :

$$
\begin{gathered}
\alpha_{o, n+1}^{*}=\alpha_{o, n}^{*}+\frac{c-\Sigma_{j} x_{j} P\left(\alpha_{o, n}^{*}-\alpha_{j}^{*}\right)}{\Sigma_{j} x_{j} P^{\prime}\left(\alpha_{o, n}^{*}-\alpha_{j}^{*}\right)} \\
\text { avec } P^{\prime}=\frac{\delta P}{\delta\left(\alpha_{o}^{*}-\alpha_{j}^{*}\right)}=-\frac{1}{\sqrt{2 \pi}} \exp \left[-\frac{1}{2}\left(\alpha_{o, n}^{*}-\alpha_{j}^{*}\right)^{2}\right] \\
y_{j}=\frac{x_{j} P\left(\alpha_{o}^{*}-\alpha_{j}^{*}\right)}{c} \\
\text { et } y_{j}^{*}=x_{j}^{*}+\sigma \sqrt{C D} i\left(\alpha_{o}^{*}-\alpha_{j}^{*}\right)
\end{gathered}
$$

On a alors

où i $\left(\alpha_{o}^{*}-\alpha_{j}^{*}\right)$ désigne l'intensité de sélection au point d'abscisse $\alpha_{o}^{*}-\alpha_{j}^{*}$. Dans le cas de la loi normale, cette quantité est aussi égale à $-\frac{\mathrm{P}^{\prime}}{\mathrm{P}}$ au point considéré.

P est calculé par interpolation polynomiale (ABRAMOWITZ \& STEGUN, 1972).

\section{4) Définitions matricielles}

Le programme de sélection défini au paragraphe IIA se traduit par des matrices ou vecteurs.

a) matrices et vecteurs de fréquences :

Ces matrices sont telles que la ligne représentè la classe d'âge (classe selon le nombre d'années révolues), la ligne 1 correspondant à la classe d'âge la plus jeune, et que la colonne représente la classe génotypique.

Toutes ces matrices ont donc $\mathrm{p}$ colonnes et sont telles que les sommes par ligne sont égales à 1 , quelles que soient la matrice et la ligne considérées.

On définit 3 matrices $\left(G_{a}, T_{a}, S_{a}\right)$ et 7 vecteurs $\left(g_{a}, p_{a}, m_{a}, t_{a}, \pi_{a}, \mu_{a}, s_{a}\right)$.

$\mathrm{G}_{\mathrm{a}}=$ matrice $(11, \mathrm{p})$ des fréquences des différents génotypes pour les 11 classes d'âge de femelles considérées l'année a (0 à 10 ans révolus c'est-à-dire 0,5 à 10,5 ans).

$\mathrm{T}_{\mathrm{a}}=$ matrice $(7, \mathrm{p})$ des fréquences des différents génotypes pour les futurs taureaux de testage ( $0-1$ an), les taureaux en testage (2-3-4-5 ans) et les taureaux nouvellement testés (6 ans), l'année a.

$S_{a}=$ matrice $(2, p)$ des fréquences des différents génotypes pour les taureaux de service, l'année $a$.

$\mathrm{g}_{\mathrm{a}}=1^{\text {re }}$ ligne de $\mathrm{G}_{\mathrm{a}}=$ vecteur ligne des fréquences pour les femelles nées l'année a.

$\mathrm{p}_{\mathrm{a}}=$ vecteur $(1, \mathrm{p})$ des fréquences pour les pères des veaux nés l'année $\mathrm{a}$.

$\mathrm{m}_{\mathrm{a}}=$ vecteur $(1, \mathrm{p})$ des fréquences pour les mères des veaux nés l'année $\mathrm{a}$.

$t_{a}=1^{\text {re }}$ ligne de $T_{a}=$ vecteur ligne des fréquences pour les mâles issus l'année a des accouplements raisonnés (pères à taureaux $\times$ mères à taureaux) programmés l'année $\mathbf{a}-1$.

$\pi_{\mathrm{a}}=$ vecteur $(1, \mathrm{p})$ des fréquences pour les pères des futurs taureaux de testage nés l'année $\mathrm{a}$.

$\mu_{\mathrm{a}}=$ vecteur $(1, \mathrm{p})$ des fréquences pour les mères des futurs taureaux de testage nés l'année $\mathrm{a}$. 
$\mathrm{s}_{\mathrm{a}}=1^{\mathrm{re}}$ ligne de $\mathrm{S}_{\mathrm{a}}=$ vecteur ligne des fréquences pour les taureaux nouvellement testés, après sélection pour le service.

b) matrices et vecteurs de niveaux génétiques

A chaque matrice ou vecteur, est associé la matrice ou le vecteur correspondant pour les niveaux génétiques.

On définit donc 3 matrices $\left(\mathrm{G}_{\mathrm{a}}^{*}, \mathrm{~T}_{\mathrm{a}}^{*}, \mathrm{~S}_{\mathrm{a}}^{*}\right)$ et 7 vecteurs $\left(\mathrm{g}_{\mathrm{a}}^{*}, \mathrm{p}_{\mathrm{a}}^{*}, \mathrm{~m}_{\mathrm{a}}^{*}, \mathrm{t}_{\mathrm{a}}^{*}, \pi_{\mathrm{a}}^{*}, \mu_{\mathrm{a}}^{*}, \mathrm{~s}_{\mathrm{a}}^{*}\right)$.

5) Relations de récurrence

a) relations de récurrence sur les matrices

a) femelles de la population commerciale

$$
\mathrm{G}_{\mathrm{a}}=\left(\begin{array}{l}
\mathrm{g}_{\mathrm{a}} \\
\dot{\mathrm{H}}_{1} \\
\dot{\mathrm{G}}_{\mathrm{a}-1}
\end{array}\right)
$$

où $H_{1}$ désigne la matrice $(10,11)$ de passage permettant de sélectionner les 10 premières lignes de $G_{a-1}$ matrice qui correspond au vieillissement de la population femelle présente l'année $\mathbf{a}-1$.

On a de même :

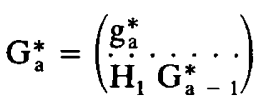

B) taureaux de testage :

$$
\mathrm{T}_{\mathrm{a}}=\left(\begin{array}{c}
\mathrm{t}_{\mathrm{a}} \\
\ddot{\mathrm{H}}_{2} \ddot{\mathrm{T}}_{\mathrm{a}-1}
\end{array}\right)
$$

où $\mathrm{H}_{2}$ désigne la matrice $(6,7)$ de passage permettant de sélectionner les 6 premières lignes de $T_{a-1}$.

De même :

$$
\mathrm{T}_{\mathrm{a}}^{*}=\left(\begin{array}{c}
\mathbf{t}_{\mathbf{a}}^{*} \\
\underset{\mathrm{H}_{2}}{\mathrm{~T}_{\mathrm{a}-1}^{*}}
\end{array}\right)
$$

$\gamma$ ) taureaux de service :

$$
\mathrm{S}_{\mathrm{a}}=\left(\begin{array}{c}
\mathrm{s}_{\mathrm{a}} \\
\ddot{H}_{3} \dot{\mathrm{S}}_{\mathrm{a}-1}
\end{array}\right)
$$

où $\mathrm{H}_{3}$ désigne la matrice $(1,2)$ de passage permettant de sélectionner la première ligne de $\mathrm{S}_{\mathrm{a}-1}$.

$$
\text { On a de même : } \quad \mathrm{S}_{\mathrm{a}}^{*}=\left(\begin{array}{r}
\mathrm{s}_{\mathrm{a}}^{*} \\
\underset{\mathrm{H}_{3}}{\mathrm{~S}_{\mathrm{a}-1}^{*}}
\end{array}\right)
$$

b) relations de récurrence sur les vecteurs :

a) Calcul de $g_{a}$ et de $g_{a}^{*}$ :

Ces vecteurs s'obtiennent respectivement à partir des formules (1) et (2), en remplaçant $\mathrm{V}$ par $\mathrm{P}_{\mathrm{a}}, \mathrm{W}$ par $\mathrm{m}_{\mathrm{a}}, \mathrm{V}^{*}$ par $\mathrm{P}_{\mathrm{a}}^{*}, \mathrm{~W}^{*}$ par $\mathrm{m}_{\mathrm{a}}^{*}$.

B) Calcul de $\mathrm{p}_{\mathrm{a}}$ et de $\mathrm{p}_{\mathrm{a}}^{*}$ :

$$
\underset{(1, p)}{p_{a}}=\underset{(1,3)}{1} \cdot\left(\frac{\beta T_{a-1,2}}{2} \cdot S_{a-1}\right)
$$


$\beta$ désigne la proportion des inséminations qui sont réalisées avec les taureaux de testage. En pratique, on prend toujours $\beta=0,15$.

$\mathrm{T}_{\mathrm{a}-1,2}$ correspond à la $2^{\mathrm{e}}$ ligne de $\mathrm{T}_{\mathrm{a}}$. Les taureaux de cette catégorie ont donc à l'année $a$ en moyenne 2,5 ans soit 30 mois. En fait, vu ce qui a été dit dans le paragraphe II, les filles de testage naissent en moyenne quand le taureau a $18+9=27$ mois. L'erreur due à la discrétisation annuelle des classes d'âge paraît minime.

$\mathrm{S}_{\mathrm{a}-1}$ correspond à la matrice de fréquence des taureaux de service l'année $\mathrm{a}-1$. Comme l'utilisation des taureaux est supposée indépendante du génotype et constante dans le temps, les fréquences correspondant aux filles issues des taureaux présents dans $\mathrm{S}_{\mathrm{a}-1}$ s'écrit bien : $\frac{1-\beta}{2} \mathrm{~S}_{\mathrm{a}-1}$

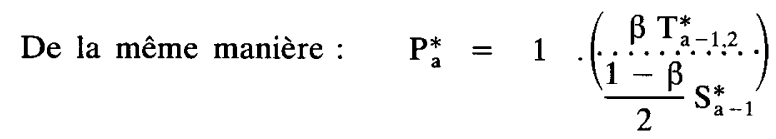

$$
(1, \mathrm{p}) \quad(1,3) \quad(3, \mathrm{p})
$$

ү) Calcul de $m_{a}$ et $m_{a}^{*}$ :

$$
\begin{aligned}
& \mathrm{m}_{\mathrm{a}}=\mathrm{F} \cdot \mathrm{H}_{4} \cdot \mathrm{G}_{\mathrm{a}-1} \\
& (1, \mathrm{p})=(1,8)(8,11)(11, \mathrm{p})
\end{aligned}
$$

où $\mathrm{F}$ désigne les fréquences des vaches du $1^{\mathrm{er}}$ vêlage au $8^{\mathrm{e}}$ vêlage.

$\mathrm{F}=0,27 \quad 0,22 \quad 0,170,13 \quad 0,09 \quad 0,06 \quad 0,04 \quad 0,02$ d'âge).

(on se place ainsi dans une hypothèse de stationnarité de la distribution des classes où $\mathrm{H}_{4}$ désigne la matrice de passage permettant de sélectionner les lignes 3 à 10 de la matrice $\mathrm{G}_{\mathrm{a}-1}$, qui représente l'état de la population femelle à l'année $\mathbf{a}-1$.

On a de la même manière :

$$
\mathrm{m}_{\mathrm{a}}^{*}=\mathrm{F} \mathrm{H}_{4} \mathrm{G}_{\mathrm{a}-1}^{*}
$$

ס) Calcul de $t_{a}$ et $t_{a}^{*}$ :

Ces vecteurs s'obtiennent à partir des formules (1) et (2) en remplaçant $\mathrm{V}$ par $\pi_{\mathrm{a}}$, $\mathrm{W}$ par $\mu_{\mathrm{a}}, \mathrm{V}^{*}$ par $\pi_{\mathrm{a}}^{*}, \mathrm{~W}^{*}$ par $\mu_{\mathrm{a}}^{*}$.

ع) Calcul de $\pi_{\mathrm{a}}$ et $\pi_{\mathrm{a}}^{*}$ :

$\pi_{a}$ et $\pi_{a}^{*}$ sont calculés d'après les formules (3) et (4) en remplaçant $\mathrm{X}$ par $T_{a-1,7}$, dernière ligne de $\mathrm{T}_{\mathrm{a}-1}$ ce qui correspond aux taureaux nouvellement testés l'année $\mathrm{a}-1$, et $\mathrm{X}^{*}$ par $\mathrm{T}_{\mathrm{a}-1,7}^{*}$ avec $\mathrm{CD}=0,85$ et $\sigma=17 \mathrm{~kg} \mathrm{MU}$ (BonaiTI \& Mocouot, 1982). Le taux de conservation est égal à $C_{p}$.

५) Calcul de $\mu_{\mathrm{a}}$ et $\mu_{\mathrm{a}}^{*}$ :

$\mu_{\mathrm{a}}$ et $\mu_{\mathrm{a}}^{*}$ sont calculés d'après les formules (3) et (4) en remplaçant X et $\mathrm{X}^{*}$ par les 6 derniers éléments de $M_{a}$ et $M_{a}^{*}$ respectivement (après division par leur somme) ce qui correspond aux vaches en quatrième lactation et plus. Dans ce cas, le coefficient de détermination $\mathrm{CD}$ est considéré comme constant et égal à 0,50 . Le taux de conservation est égal à $C_{M}$. 
$\eta)$ Calcul de $s_{a}$ et $s_{a}^{*}$ :

$s_{a}$ et $s_{a}^{*}$ sont calculés de la même manière que $\pi_{a}$ et $\pi_{a}^{*}$ mais en prenant un taux de conservation $C_{s}$, différent de $C_{p}$.

\section{Problèmes de calcul liés aux importations Holstein}

Nous avons admis dans la programmation des calculs la possibilité de substituer à tout moment certaines matrices ou vecteurs calculés dans le cadre du modèle (notation générale X) par des valeurs $\mathbf{X}$, provenant de l'observation ou de considérations $a$ priori. Ces matrices ou vecteurs ne concernent que les taureaux de testage ou de service, catégories très directement influencées par les décisions d'importation.

En effet, depuis 1968, les importations d'animaux Holstein, tout particulièrement au niveau des taureaux de testage ont été continues et telles que la proportion de gènes Holstein prédite dans le cadre du modèle a été systématiquement inférieure à la proportion réelle. Il a paru par ailleurs indispensable de prévoir la possibilité d'étude de scénarios a priori pour le futur.

Le remplacement de matrices ou vecteurs de fréquences ne pose pas de problèmes particuliers. Ce n'est pas le cas pour les matrices de niveaux génétiques de production laitière (matière utile) pour des raisons que l'on verra au paragraphe suivant.

La base de calcul pour les niveaux génétiques laitiers est le niveau génétique moyen des plus vieilles femelles frisonnes (nées en 1956) présentes en 1967, année précédant l'introduction de la Holstein dans notre modèle.

\section{Animaux nés entre 1956 et 1967}

On suppose que le schéma de sélection décrit au paragraphe IIA est en application depuis une longue date et que le progrès génétique est dans sa phase asymptotique c'est-à-dire linéaire. Le progrès génétique annuel est alors de 2,8 $\mathrm{kg} \mathrm{MU}$ dans ces conditions. Les niveaux génétiques des différentes catégories d'animaux frisons se situent sur des droites parallèles, dont les écarts peuvent être facilement calculés à partir des paramètres concernant les 4 voies de transmission du progrès génétique.

L'utilisation de notre programme de calcul dans l'hypothèse d'une absence d'introduction de la Holstein conduit effectivement à une évolution linéaire des niveaux génétiques conforme aux valeurs attendues suivant la formule de RENDEL \& ROBERTSON (1950).

\section{Taureaux de testage nés entre 1968 et 1980 , et taureaux de service utilisés entre 1968 et 1980}

Pour les séries de testage les plus récentes, les résultats d'indexation sur descendance ne sont pas connus. Dans ce cas, l'observation prise en compte dans les calculs correspond non à l'index sur descendance mais à un index généalogique tenant compte du père et de la mère.

La connaissance des index des taureaux n'est pas suffisante à une intégration totalement satisfaisante au modèle. En effet, dans celui-ci, les années de naissance et 
les types d'animaux (testage, service) sont parfaitement individualisés, alors qu'ils ne sont pas les critères qui ont servi à la détermination des bases fixes de calcul, tant française qu'américaine :

- la base fixe française de calcul des index correspond au niveau génétique moyen de tous les taureaux pères des vaches en lactation en 1975 (Poutous et al., 1981) : taureaux de service, taureaux de testage, taureaux de monte naturelle;

- la base fixe américaine correspond au niveau moyen de tous les taureaux pères des vaches en lactation de 1960 à 1974 (DiCKINSON, 1980).

Par ailleurs, la valeur relative de ces 2 bases fixes est encore mal connue mais elle devrait se situer aux alentours de $30 \mathrm{~kg}$ MU (Duplan, 1980 ; Duplan \& Bougler, 1982). La base fixe américaine correspond à des lactations de 1968 en moyenne. C'est approximativement l'année où les taureaux de testage nés en 1962 ont été indexés. C'est pour cette raison que nous avons paramétré la différence de niveau génétique $\left(\Delta_{62}\right)$ entre les taureaux de la base américaine et les taureaux de testage frisons nés en 1962. Les rapports entre $\Delta_{62}$ et la différence de niveaux entre taureaux de testage homologues dans les 2 pays sont loin d'être clairs: la base américaine intègre des années de naissance très disparates, ce qui permet une interférence non seulement de l'évolution des niveaux génétiques mais de l'évolution des effectifs ; elle intègre des pressions de sélection sans doute très variables au cours du temps et des taureaux de monte naturelle, en pondération inconnue. $\Delta_{62}$ n'est donc pas une différence de niveau génétique en 1962 : il faut insister sur ce point.

Malheureusement, la signification de la base française par rapport à nos taureaux de testage frisons de 1962 est aussi peu claire pour les mêmes raisons. Il est néanmoins probable que cette base correspond à un niveau supérieur à celui des taureaux de testage nés en 1962 (les taureaux concernés sont plus jeunes et plus sélectionnés). La différence entre bases fixes ayant été estimée à $30 \mathrm{~kg}$ de $\mathrm{MU}$, la valeur de $\Delta_{62}$ pourrait se situer aux alentours de $35 \mathrm{~kg}$ MU.

\section{Taureaux nés postérieurement}

Il s'agit là essentiellement du futur pour lequel différents scénarios peuvent être envisagés. Nous reviendrons sur ce point pour exposer les modalités du paramétrage correspondant.

\section{Facteurs de variation étudiés}

\section{Nombre de types génétiques}

Les 2 valeurs comparées sont 10 et 30 types génétiques. Cette dernière valeur correspond au maximum possible compte tenu des partitions mémoire dont nous disposions sur l'ordinateur et de la structure des programmes de calcul. Il aurait été possible d'aller plus loin mais cela aurait nécessité une refonte complète des techniques de programmation utilisées.

\section{Intensité de l'importation des taureaux Holstein de testage dans le futur}

Nous étudions 2 hypothèses extrêmes qui encadreront à notre avis la réalité. 
Dans l'hypothèse A, toute importation de taureaux Holstein ou de semence Holstein est arrêtée à partir de 1982. Les reproducteurs mâles et femelles sont utilisés indépendamment de leur génotype, pourvu qu'ils satisfassent au critère de sélection d'après les modalités décrites dans le schéma de sélection de référence. De cette manière, on évalue le degré de diffusion des gènes Holstein entraîné par la politique passée qui correspond quantitativement aux tableaux 1 et 2 .

Dans l'hypothèse $B$, on se situe à l'autre extrême où non seulement les seuls taureaux mis au testage sont des taureaux Holstein mais où ceux-ci sont importés d'Amérique du Nord. L'hypothèse $B$ correspond donc à une situation de diffusion maximale des gènes Holstein, le seul frein étant le faible rythme de reproduction des bovins.

3. L'écart initial du niveau génétique entre la population Frisonne française et la population Holstein nord-américaine

Ce paramètre n'est pas reflété dans les index des taureaux importés mais est très important, car il influence la diffusion ultérieure des gènes correspondants. Nous donnons donc 3 valeurs possibles au paramètre $\Delta_{62}$ (écart génétique entre les taureaux de la base américaine et les taureaux frisons nés en 1962) : 25, 35, $45 \mathrm{~kg}$ MU. La valeur centrale nous paraît être la plus probable. En effet, les taureaux frisons nés en 1962 ont une valeur génétique inférieure à ceux de la base française, parce que plus anciens, cette base se situant elle-même à environ $30 \mathrm{~kg}$ MU au-dessous de la base américaine, comme il a été dit auparavant.

\section{L'efficacité des programmes de sélection en France et aux Etats-Unis}

Les schémas de base étudiés sont :

- pour la France : le schéma décrit au paragraphe IIA ;

- pour les Etats-Unis : un schéma tel que l'évolution génétique annuelle des taureaux provenant de ce pays correspond à $3 \mathrm{~kg}$ MU. Cette valeur est très supérieure aux valeurs constatées actuellement en Amérique du Nord (Powel et al., 1977, 1980). Cependant, elle paraît être la plus réaliste, car elle tient compte de la sévérité croissante des importateurs pour le choix des taureaux et de l'intensification très probable de la sélection laitière aux Etats-Unis (apport de techniques nouvelles telles que le transfert d'œufs, naissance de la concurrence européenne).

Nous introduirons néanmoins une variante pour chacun des pays, variante intervenant dans le futur:

- pour la France : le schéma adopté conduirait en population fermée à un progrès génétique annuel de $3,56 \mathrm{~kg} \mathrm{MU}\left(\mathrm{C}_{\mathrm{p}}=\mathrm{C}_{\mathrm{M}}=0,01 ; \mathrm{C}_{\mathrm{S}}=0,25\right)$;

- pour les Etats-Unis : le progrès génétique annuel sur les taureaux importés se situe au niveau modeste de $2 \mathrm{~kg}$ MU.

\section{E. Critères d'expression des résultats}

Les calculs sont effectués en prenant l'année 1967 comme année initiale et en considérant une durée de 30 ans. 
Nous exprimons les résultats de manière allégée pour faire apparaître ce qui est à notre avis essentiel. Seuls les résultats de 1983 à 1997, exprimés par période de 2 ans pour les veaux nés aux années correspondantes sont exprimés, bien que toutes les catégories d'animaux soient impliquées dans les calculs. Sur ces veaux, on s'intéresse d'une part à la fréquence des gènes Holstein (en ignorant la distribution des différents types génétiques) et d'autre part à leur niveau génétique pour la production de matière utile, exprimé en écart à celui qui aurait été obtenu par sélection dans une population frisonne constamment fermée.

Nous avons choisi de ne pas détailler au-delà de l'année 1997, dans 14 ans. Quand on observe l'évolution des techniques dans différents domaines, il est très possible que les programmes de sélection chez les bovins laitiers soient alors très différents de ceux qui sont décrits ici. Une estimation ponctuelle pour l'année 2017 (20 ans plus tard) est néanmoins donnée dans les tableaux de résultats, de manière à détecter d'éventuelles erreurs de conclusions.

\section{Résultats et discussion}

\section{A. Résultats obtenus pour les programmes de sélection de référence}

Le programme de sélection français est celui décrit au paragraphe IIA et le progrès génétique annuel sur les taureaux importés d'Amérique du Nord est supposé égal à $3 \mathrm{~kg}$ MU. Les 3 facteurs de variation étudiés ici sont les 3 premiers facteurs décrits en IID.

Les résultats concernant les fréquences de gènes Holstein figurent au tableau 3 et ceux concernant les niveaux génétiques au tableau 4.

Pour les 2 types de résultats, il est clair que la représentation de tous les types génétiques possibles en seulement 10 classes est largement suffisante pour l'analyse de l'effet des autres paramètres, à condition que l'ensemble des animaux n'ait pas atteint un degré de croisement très élevé (hypothèse B après 1990), avec une fréquence élevée de Holstein purs ou situés dans la dernière classe de holsteinisation. En effet, un animal Holstein pur se voit attribuer artificiellement 5 p. 100 de gènes européens dans le cas de 10 classes alors que cette valeur n'est plus que de 1,7 p. 100 dans le cas de 30 classes. Dans ces situations extrêmes, l'utilisation d'un petit nombre de classes conduit à une certaine sous-estimation de la vitesse de diffusion des gènes Holstein.

Les résultats obtenus avant 1990 pour les 2 hypothèses (A et B) et après 1990 pour l'hypothèse $A$ ne diffèrent pratiquement pas suivant le nombre de classes génétiques retenues. En effet, les animaux concernés sont à des degrés intermédiaires de croisement. Les erreurs d'appréciation sur chacun d'eux peuvent être soit des surestimations du degré de sang Holstein soit des sous-estimations, l'erreur globale, fonction algébrique des erreurs individuelles, est pratiquement nulle et donc indépendante de la précision de la construction des classes génétiques.

On note que les fréquences de gènes Holstein obtenues en 1983 (fréquences actuelles) sont déjà considérables (de l'ordre de 35 p. 100). Ce niveau est dans tous les cas destiné à augmenter encore. Suivant les hypothèses, le niveau atteint en 1997 varie approximativement de 40 p. 100 à 85 p. 100 . Dans le cas de l'hypothèse $\mathrm{A}$, les niveaux atteints en 1997 (de l'ordre de 50 p. 100) sont bien entendu inférieurs à ceux obtenus lors 
TABLEAU 3

Fréquence (\%) des gènes Holstein chez les veaux de l'année dans différentes situations.

Holstein (\%) gene frequency in the calf population according to various situations.

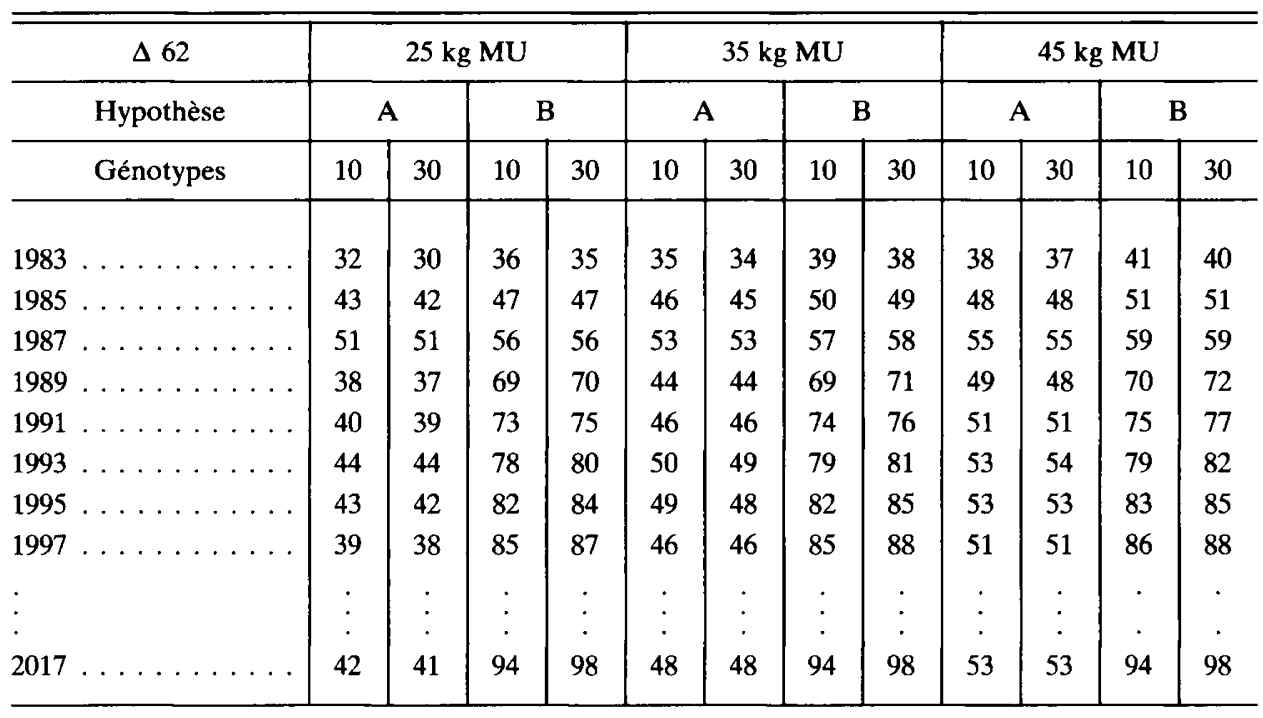

Hypothèse $\mathrm{A}=$ arrêt des importations Holstein; Hypothèse $\mathrm{B}=$ absorption maximale avec la Holstein ; $\Delta 62$ = écart de niveau génétique entre les taureaux Pie-Noirs nés en France en 1962 et les taureaux de la base fixe de calcul des indices américains.

Hypothesis $A=$ stopping the Holstein importations; Hypothesis $B=$ maximum grading-up by Holstein young bulls; $\Delta 62=$ genetic gap between the French Friesian bulls born in 1962 and the bulls of the fixed basis used for sire evaluation in the U.S.

d'une holsteinisation maximale (de l'ordre de 85 p. 100). Les niveaux atteints en cas de fermeture des frontières (hypothèse $\mathrm{A}$ ) dépendent évidemment de l'écart génétique initial entre populations et donc du paramètre $\Delta_{62}$ avec lequel cet écart est en corrélation (bien qu'il ne le représente pas) : la fréquence Holstein augmente d'environ 0,5 p. 100 par $\mathrm{kg}$ supplémentaire de $\Delta_{62}$. Les niveaux atteints lors de l'hypothèse $\mathrm{B}$ dépendent beaucoup moins des écarts génétiques initiaux : la fréquence Holstein n'augmente que d'environ 0,15 p. 100 par $\mathrm{kg}$ supplémentaire de $\Delta_{62}$. L'ensemble de ces résultats peut être considéré comme évident d'un point de vue qualitatif, l'intérêt du modèle étant précisément de quantifier l'effet de ces différents paramètres.

L'apport du croisement Holstein est dans tous les cas positif en ce qui concerne l'évolution génétique de la production de matière utile.

La politique de holsteinisation maximum (B) est plus bénéfique que celle de la fermeture des frontières après environ 15 ans d'immigration libre $(A)$. L'ampleur de ce bénéfice dépend très largement du paramètre $\Delta_{62}$. En effet, quand cette valeur est faible ( $25 \mathrm{~kg} \mathrm{MU}$ ) les 2 politiques n'apportent pratiquement rien de plus qu'un programme de sélection en race pure. Pour les autres valeurs, les gains peuvent être substantiels, entre 6 et $20 \mathrm{~kg}$ MU : ce qui correspond respectivement à 2 et à 7 années de sélection en race pure. 


\section{TABLEAU 4}

Valeur génétique moyenne des animaux du tableau 3, exprimée en $\mathrm{kg}$ de matière utile et en écart à la valeur obtenue sans introduction de la Holstein (schéma de sélection décrit au paragraphe IIA2).

Average breeding value for the animals of the previous table, expressed in $\mathrm{kg}$ "valuable solids" and in deviation to an hypothetic value obtained without introducing Holstein [selection scheme described in paragraph (IIA2)].

\begin{tabular}{|c|c|c|c|c|c|c|c|c|c|c|c|c|c|}
\hline & $\Delta 62$ & \multicolumn{4}{|c|}{$25 \mathrm{~kg} \mathrm{MU}$} & \multicolumn{4}{|c|}{$35 \mathrm{~kg} \mathrm{MU}$} & \multicolumn{4}{|c|}{$45 \mathrm{~kg} \mathrm{MU}$} \\
\hline & Hypothèse & \multicolumn{2}{|c|}{ A } & \multicolumn{2}{|c|}{ B } & \multicolumn{2}{|c|}{ A } & \multicolumn{2}{|c|}{ B } & \multicolumn{2}{|c|}{ A } & \multicolumn{2}{|c|}{ B } \\
\hline & Génotypes & 10 & 30 & 10 & 30 & 10 & 30 & 10 & 30 & 10 & 30 & 10 & 30 \\
\hline 1983 & $\ldots$ & 2 & 1 & 2 & 2 & 5 & 5 & 5 & 5 & 9 & 9 & 10 & 10 \\
\hline 1985 & 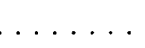 & 1 & 1 & 1 & 2 & 6 & 6 & 6 & 6 & 11 & 11 & 12 & 12 \\
\hline 1987 & $\therefore$ & 2 & 2 & 2 & 2 & 7 & 7 & 8 & 8 & 14 & 13 & 14 & 14 \\
\hline 1989 & 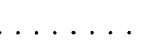 & 2 & 2 & 2 & 2 & 6 & 6 & 10 & 9 & 11 & 11 & 17 & 17 \\
\hline 1991 & & 2 & 2 & 2 & 2 & 6 & 6 & 10 & 10 & 12 & 12 & 18 & 18 \\
\hline 1993 & $\ldots \ldots$ & 1 & 1 & 0 & 1 & 6 & 6 & 8 & 8 & 12 & 12 & 17 & 16 \\
\hline 1995 & $\because$ & 2 & 2 & 1 & 4 & 7 & 7 & 10 & 10 & 12 & 12 & 19 & 19 \\
\hline 1997 & 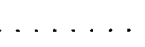 & 2 & 2 & 2 & 1 & 6 & 6 & 11 & 10 & 12 & 12 & 20 & 19 \\
\hline : & & . & . & - & . & . & . & . & . & . & : & : & : \\
\hline 2017 & & 1 & 1 & 5 & 5 & 6 & 6 & 15 & 15 & 12 & 11 & 25 & 25 \\
\hline
\end{tabular}

Hypothèse $\mathrm{A} ; \mathrm{B} ; \Delta 62$ : voir la signification au tableau 3.

Hypothesis $A ; B ; \Delta 62$ : see at table 3 for explanation.

\section{B. Résultats obtenus en faisant varier les programmes de sélection}

Ces résultats sont rassemblés aux tableaux 5 et 6 . Ils correspondent à 10 types génétiques et à la valeur 35 pour le paramètre $\Delta_{62}$.

Les résultats du tableau 5 concernant les fréquences de gènes Holstein montrent qu'une intensification des programmes de sélection laitière en France (passage du progrès génétique asymptotique annuel en race pure de $2,8 \mathrm{~kg} \mathrm{MU}$ à $3,6 \mathrm{~kg}$ MU soit un accroissement d'environ 30 p. 100 , ce qui est très important) n'a que peu d'incidence sur les fréquences de gènes Holstein à long terme, dans les 2 hypothèses $\mathrm{A}$ et $\mathrm{B}$. Cette relative rigidité s'explique à notre sens par les particularités de calcul introduites (introduction de valeurs « observées » en fréquence et en niveaux génétiques pour un assez grand nombre d'années initiales pour les hypothèses $\mathrm{A}$ et $\mathrm{B}$; valeurs de fréquences $a$ priori dans le cas des années finales de l'hypothèse $\mathrm{B}$ ). L'efficacité future des programmes de sélection en Amérique du Nord n'a évidemment aucune incidence sur les fréquences. Nous l'avons d'ailleurs vérifié par le calcul.

Les résultats du tableau 6 indiquent cependant que le bénéfice du croisement Holstein, en terme de niveau génétique laitier, dépend largement de l'efficacité des programmes de sélection en France et en Amérique du Nord. Une politique continue d'importation de taureaux Holstein purs issus d'un schéma de sélection peu efficient, 


\section{TABleau 5}

Fréquences des gènes Holstein (\%) chez les veaux de l'année en fonction de l'intensité des programmes de sélection en France et aux U.S.A.

Holstein gene frequencies (\%) in the calf population according to the intensity of the selection programmes in France and in the U.S.

\begin{tabular}{|c|c|c|c|c|}
\hline $\begin{array}{c}\text { Programme de sélection } \\
\text { en France }\end{array}$ & \multicolumn{2}{|c|}{$\begin{array}{l}\text { Référence (IIA) } \\
(2,8 \mathrm{~kg} \mathrm{MU} / \mathrm{an})\end{array}$} & \multicolumn{2}{|c|}{$\begin{array}{c}\text { Intensif } \\
(3,6 \mathrm{~kg} \mathrm{MU} / \mathrm{an})\end{array}$} \\
\hline $\begin{array}{l}\text { Progrès génétique annuel } \\
\text { aux U.S.A. }\end{array}$ & \multicolumn{2}{|c|}{$3 \mathrm{~kg} \mathrm{MU}$ ou $2 \mathrm{~kg} \mathrm{MU}$} & \multicolumn{2}{|c|}{$3 \mathrm{~kg}$ MU ou $2 \mathrm{~kg} \mathrm{MU}$} \\
\hline Hypothèse sur les importations & A & B & A & B \\
\hline 1983 & 35 & 39 & 37 & 40 \\
\hline$\ldots \ldots \ldots \ldots \ldots$ & 46 & 50 & 48 & 51 \\
\hline$\ldots \ldots$ & 53 & 57 & 54 & 58 \\
\hline 1989 & 44 & 69 & 46 & 70 \\
\hline 1991 & 46 & 74 & 48 & 75 \\
\hline 1993 & 50 & 79 & 51 & 79 \\
\hline 1995 & 49 & 82 & 50 & 83 \\
\hline $1997 \ldots \ldots \ldots \ldots \ldots$ & 46 & 85 & 48 & 86 \\
\hline : & : & : & . & : \\
\hline & . & . & & . \\
\hline 2017 & 48 & 94 & 50 & 94 \\
\hline
\end{tabular}

peut être une erreur et mener à long terme (dans au moins 10 ans) à des niveaux génétiques laitiers équivalents ou même inférieurs à ceux obtenus après un arrêt total des importations et intensification des pressions de sélection laitière dans le programme national. En fait, l'optimisation de l'introduction des gènes Holstein en vue du progrès génétique laitier à long terme constitue un sujet différent de celui abordé dans cet article.

\section{Conclusion}

Les grandes lignes des résultats de notre étude apparaissent clairement : le processus de diffusion des gènes Holstein, bien qu'il ait atteint déjà un niveau élevé (fréquence probable de 35 p. 100 chez les veaux nés en 1983), ne fait en réalité que commencer. Il $\mathrm{y}$ a tout lieu de penser que dans 15 ans le niveau atteint sera environ du double. Concrètement, cela veut dire que la Française Frisonne sera devenue une variété de Holstein avec une fraction très nettement minoritaire de gènes Frisons. Ceci ne peut manquer d'avoir des conséquences zootechniques considérables, favorables et défavorables, au moment même où la race Pie Noir Française poursuit son avance démographique en France (Duplan \& Bougler, 1980).

Il apparaît également que tout retour en arrière est dès à présent impossible. La migration continuelle de gènes Holstein pendant 15 ans est telle que la fréquence potentielle au niveau de la population française est de l'ordre de $50 \mathrm{p}$. 100 . Ce fait apparaissait déjà lors d'une étude préliminaire antérieure (COLLEAU, 1978). Il est tout 


\section{Tableau 6}

Valeur génétique moyenne des animaux du tableau 5 exprimée en $\mathrm{kg}$ de matière utile et en écart à la valeur obtenue sans introduction de la Holstein.

Average breeding value for the animals of preceeding table, expressed in $\mathrm{kg}$ "valuable solids" and in deviation from an hypothetic value obtained without introducing Holstein.

\begin{tabular}{|c|c|c|c|c|c|c|c|c|}
\hline $\begin{array}{c}\text { Programme de sélection } \\
\text { en France }\end{array}$ & \multicolumn{4}{|c|}{$\begin{array}{c}\text { Référence } \\
(2,8 \mathrm{~kg} \text { MU/an) }\end{array}$} & \multicolumn{4}{|c|}{$\begin{array}{c}\text { Intensif } \\
(3,6 \mathrm{~kg} \mathrm{MU} / \mathrm{an})\end{array}$} \\
\hline $\begin{array}{l}\text { Progrès génétique annuel } \\
\text { aux U.S.A. }\end{array}$ & \multicolumn{2}{|c|}{$3 \mathrm{~kg} \cdot \mathrm{MU}$} & \multicolumn{2}{|c|}{$2 \mathrm{~kg} \mathrm{MU}$} & \multicolumn{2}{|c|}{$3 \mathrm{~kg} \mathrm{MU}$} & \multicolumn{2}{|c|}{$2 \mathrm{~kg} \mathrm{MU}$} \\
\hline Hypothèses sur les importations & A & B & A & B & A & B & A & B \\
\hline$\ldots \ldots \ldots \ldots \ldots \ldots$ & 5 & 5 & 5 & 5 & 5 & 6 & 5 & 6 \\
\hline $1985 \ldots \ldots \ldots \ldots \ldots \ldots$ & 6 & 6 & 6 & 6 & 6 & 7 & 6 & 6 \\
\hline $1987 \ldots \ldots \ldots \ldots \ldots \ldots$ & 7 & 8 & 7 & 7 & 7 & 7 & 7 & 7 \\
\hline $1989 \ldots \ldots \ldots \ldots \ldots \ldots$ & 6 & 9 & 6 & 8 & 6 & 7 & 6 & 5 \\
\hline $1991 \ldots \ldots \ldots \ldots \ldots \ldots$ & 6 & 10 & 6 & 8 & 7 & 7 & 7 & 5 \\
\hline$\ldots \ldots \ldots \ldots \ldots$ & 6 & 8 & 6 & 5 & 6 & 5 & 6 & 1 \\
\hline$\ldots \ldots \ldots$ & 7 & 10 & 7 & 5 & 7 & 6 & 7 & 0 \\
\hline $1997 \ldots$ & 6 & 10 & 6 & 4 & 7 & 5 & 7 & -2 \\
\hline . & : & . & : & : & : & : & : & : \\
\hline 2017 & 6 & 15 & 6 & -10 & 7 & 1 & $\dot{7}$ & -24 \\
\hline
\end{tabular}

à fait probable que dans bon nombre de pays européens de l'Ouest, les niveaux actuels et potentiels de gènes Holstein sont comparables à ceux de la France. Les conséquences zootechniques et donc économiques de ce qu'on pourrait appeler « holsteinisation » des races frisonnes européennes seront sans doute importantes.

D'un point de vue analytique, la description simplifiée de la diffusion des gènes Holstein sous forme de catégories discrètes paraît bien adaptée. C'est le point qui nous paraît le plus positif. Par ailleurs, nous sommes conscients que les programmes de sélection décrits sont très schématiques alors que dans la réalité, ils sont plus complexes, portent sur plusieurs caractères, fluctuent dans le temps. La lourdeur du modèle aurait été plus grande de même que celle de la programmation des calculs. Nous désirions seulement disposer dans un premier temps d'un ordre de grandeur de la vitesse de diffusion des gènes Holstein. Nous signalerons enfin que toute tentative de prévision fine est compliquée par le problème de la signification des bases d'indexation utilisées en France et en Amérique du Nord et de l'estimation des différences entre bases. Ces paramètres ont un impact majeur sur l'expression des résultats.

Reçu le 13 juillet 1983. Accepté le 15 février 1984.

\section{Remerciements}

Nous remercions les lecteurs de la revue pour leur examen approfondi du manuscrit. Nous adressons également nos remerciements à $M$. $P$. BoulANGER et à $\mathbf{M}^{\text {me }} \mathbf{P}$. LE MEZEC, de l'Institut Technique de l'Elevage Bovin, qui nous ont fourni les statistiques nécessaires. 


\section{Références bibliographiques}

Abramowitz M., Stegun I.A., 1972. Handbook of mathematical functions. Dover Publications, Inc., New York, $1046 \mathrm{p}$.

BECH ANDERSEN B., 1982a. Danish breeding strategies for milk and beef traits in dairy and dual purpose cattle breeds. In : MORE o'Ferrall (ed.), Beef production from different dairy breeds and dairy beef crosses (Curr. Top. Vet. Med. Anim. Sci., 21). Martinus Nijhoff, The Hague, 29-37.

Bech ANDERSEN B., 1982b. Comparison of Danish Friesian, Holstein Friesian and Red Danish for beef production. In : MORE O'FERRALL (ed.), Beef production from different dairy breeds and dairy beef crosses (Curr. Top. Vet. Med. Anim. Sci., 21). Martinus Nijhoff, The Hague, 268-278.

Bonaiti B., MocQuot J.C., 1982. Etudes sur la production laitière des bovins. IV. Paramètres génétiques en première lactation. Ann. Génét. Sél. Anim., 14, 161-176.

Bougler J., DuPlan J.M., 1981. L'évolution des cheptels bovins laitiers français et ouest-européens. In : JARRIGE R., TiRel J.C. (ed.), La production laitière française. Evolution récente et pers. pectives. I.N.R.A. Publ., Versailles, 27-48.

Colleau J.J., 1978. «Holsteinisation» de la population Pie Noire française (prévision de la fréquence des gènes Holstein dans les 20 années à venir). Bull. Tech. C.R.Z.V. Theix-I.N.R.A., $34,45-50$.

Colleau J.J., Tanguy D., Boulanger P., Le Mezec P., 1982. Prediction of diffusion of Holstein genes within the French Friesian ponulation. 2nd World Congress on Genetics applied to Livestock Production, Madrid, 4-8 october 1982, 8, 226-233, Editorial Garsi, Madrid.

DiCKINSON F.N., 1980. Alternative genetic bases for sire summaries and cow indexes. J. Dairy Sci., 63, 1361-1365.

DuBIS J.M., 1979. Quelles vaches laitières pour demain? Comparaison de différents types. Institut Technique de l'Elevage Bovin $\mathbf{n}^{\circ} 79063$, M.N.E., Paris, 19 pp. ronéotées.

Duplan J.M., 1980. La valeur génétique des taureaux dans les pays d'élevage laitier développé. Institut Technique de l'Elevage Bovin. Section Amélioration Génétique, no 1049, M.N.E., Paris.

ERnst E., Langlet J.F., Martin H., 1973. Vergleichende Untersuchungen an deutschen Schwarzbunten, Holstein-Friesians sowie den Kreuzungen aus beiden Populationen. Schriftenr. Agrarwiss. Fak. Univ. Kiel, 50, 1-160.

Jongeling C., Niedhaves W., 1979. Die Entwicklung der Fortpflanzungsleistung niedersächsischer Besamungsbullen in Abhängigheit vom Holstein Friesian Genanteil. Der Tierz., 30, 506-508.

Journet M., Hoden A., Geay Y., Lienard G., 1973. Comparaison entre animaux Pie Noir de type Holstein Canadien et de type Frison Français. Bull. Tech. C.R.Z.V. Theix-I.N.R.A., 12, 13-25.

Krausslich H., 1974. Comparison of daughters of Holstein-Friesian and German Black and White bulls under different environmental conditions. Proceedings of the Working Symposium on Breed Evaluation and Cross Experiments with Farm Animals, 15-21 September 1974. 1.V.O. (Ed.), 199-207, Zeist.

LeROY P., 1977. Influence du croisement Holstein-Friesian $\times$ Pie Noir $(\mathrm{HF} \times \mathrm{PN})$ sur la production laitière, la production de viande et la fertilité. Ann. Med. Vet., 121, 159-167.

Milk Marketing Board, 1982. The Confederation of United Kingdom Milk Marketing Boards. (Ed.), Dairy facts and figures 1981. Thames Ditton, Surrey, U.K.

Ministère Canadien de l'Agriculture, 1980. Food Production and Marketing Branch. Animal Production Division. (Ed.) Dairy sire appraisal $n^{\circ} 42$.

Minkema D., 1974. An experiment on crossbreeding between Holstein-Friesian bulls and Dutch Friesian cows. Proceedings of the Working Symposium in Breed Evaluation and Cross Experiments with Farm Animals, 15-21 september 1974, I.V.O. (Ed.), 207-216, Zeist.

Mocouot J.C., BonarTi B., 1981. Composition du lait : paramètres génétiques et sélection. Elevage et Insémination, 186, 9-16.

MOKHTAR I., 1979. Wechselbeziehungen zwischen Standortintensität und genetischer Leistungsveranlagung untersucht an Einkreuzungsstufen von Holstein-Friesian in der Niedersachsischen Schwarzbuntzucht. Dissertation, Universität Göttingen. 
Oldensroek J.K., 1974. Comparison of North American Friesian, Dutch Friesian and Dutch Red and White cattle. Proceedings of the Working Symposium on Breed Evaluation and Crossing Experiments with Farm Animals, 15-21 september 1974, I.V.O. (Ed.), 217-225, Zeist.

OLDENBROEK J.K., 1979. Additive genetic, heterosis and maternal effects on production traits in a crossing experiment between Dutch Friesian and Holstein Friesian cattle. 30th Annual Meeting of the E.A.A.P., July 1979, Harrogate (England).

OLDENBROEK J.K., 1980. Breed and crossbreeding effects in a crossing experiment between Dutch Friesian and Holstein Friesian cattle. Livest. Prod. Sci., 7, 235-241.

OldenBroek J.K., 1982. Meat production from Holstein Friesians in comparison to Dutch Friesians and Dutch Red and Whites. In : MORE O'FERRALl (ed.), Beef production from different dairy breeds (Curr. Top. Vet. Med. Anim. Sci., 21), 45-52, Martinus Nijhoff, The Hague.

Oldenbroek J.K., 1983a. A comparison of Holstein Friesians, Dutch Friesians and Dutch Red and Whites. I. Production characteristics. Livest. Prod. Sci., 11, 69-81.

Oldenbroek J.K., 1983b. A comparison of Holstein Friesians, Dutch Friesians and Dutch Red and Whites. II. Economic synthesis with different systems for milk and meat. Livest. Prod. Sci., 11, 83-90.

Philipsson J., Hansson I., Henningsson T., 1981. Growth rate and carcass characteristics of different strains of Friesian cattle. 32nd Annual Meeting of the E.A.A.P., 31 August-3 September 1981, Zagreb, Yugoslavia.

PolitiEK R.D., 1974. The comparison of Friesians from different origin. I. Comparison of the production of Dutch Friesians randomly sampled within two breeding districts and herd levels. Z. Tierz. Züchtungsbiol., 91, 1-10.

Politiek R.D., Vos H., Korver S., 1982. Comparison of Friesian cattle from different origins. II. Milk production in two subpopulations from the Netherlands and progeny of Dutch Friesian, Holstein Friesian and British Friesian proven bulls. Z. Tierz. Züchtungsbiol., 99, 272-285.

Poutous M., Briend M., Calomiti S., Doan D., Felgines C., Steier G., 1981. Méthode de calcul des index laitiers. Bull. Tech. Ing. Serv. Agric., 361, 433-446.

Powell R.L., NoRman H.D., Dickinson F.N., 1977. Trends in breeding value and production. $J$. Dairy Sci., 60, 1316-1326.

Powell R.L., Norman H.D., Dickinson F.N., 1980. Genetic means and trends of dairy sires in the United States. J. Dairy Sci., 63, 1455-1461.

REGIS R., 1979. Comparaison de l'aptitude à la production de veaux de boucherie de différents types génétiques Pie Noirs. Institut Technique de l'Elevage Bovin, 18 pages ronéotées, M.N.E., Paris.

REGIS R., 1980. Comparaison de l'aptitude à la production de taurillons de différents types génétiques Pie Noirs. Institut Technique de l'Elevage Bovin, no 80034, M.N.E., Paris.

ReNDEl J.M., RoBerTson A., 1950. Estimation of genetic gain in milk yield by selection in a close herd of dairy cattle. J. Genet., 50, 1-8.

Southgate J.R., 1982. A note on the comparison of Holsteins and Friesians for growth, feed efficiency and carcass traits. In : MORE O'FERRALL (ed.), Beef production from different dairy breeds and dairy beef crosses (Curr. Top. Vet. Med. Anim. Sci., 21), 37-44, Martinus Nijhoff, The Hague.

Stolzman M., Jasiorowski H., Reklewski Z., Zarnecki A., 1981. Provisional results on milk and fattening performance of F1 Friesian strain crosses in the field. 32nd Annual Meeting of the E.A.A.P., 31 August-3 September 1981, Zagreb (Yugoslavia).

Turton J.D., 1981. Crossbreeding of dairy cattle : a selection review. Anim. Breed. Abstr., 49, 293-300.

Witt M., Andreae U., Huth F.W., Kallweit E., Pfleiderer U.E., Rappen W., von Schutzbar W., Werhahn E., Moseler W., Selhavsen D., 1971. Einfluss der Kreuzung von Vatertieren der Holstein-Friesian. Rasse mit deutschen schwarzbunten Kühen auf Korperentwicklung, Milchleistung und Musckelbildungsvermögen. Schriftenr. Max-Planck-Inst. Tierz. Tierernähr, Mariensee, 56, 1-92. 